\title{
Poli (Metil Azoteto de Glicidila) - GAP. I. Síntese e Caracterização
}

\author{
Sandro P. Ribeiro \\ Seção de Engenharia Química, IME \\ David G. Santiago \\ Centro Tecnológico do Exército, CTEx \\ Ardson dos S. Vianna Jr. \\ Departamento de Engenharia Química, Escola Politécnica, USP
}

\begin{abstract}
Resumo: O poli (metil azoteto de glicidila) - GAP - é um material energético que pode ser utilizado como aglutinante (binder) e como plastificante energético em compostos explosivos e propulsores de foguetes. Neste trabalho, foi abordada a síntese do (GAP) através da conversão direta da epicloridrina $(\mathrm{ECH})$ a GAP. Os reagentes utilizados foram azida de sódio, epicloridrina e vários álcoois extensores de cadeias, o etanodiol, o 1,4-butanodiol, o dietilenoglicol e o glicerol. Alguns parâmetros de operação foram avaliados, como o tempo de reação, a proporção entre os reagentes, dois tipos de solvente e a ordem de adição dos reagentes. A variável observada para a análise foi a massa molecular do GAP. Todos os materiais sintetizados também foram caracterizados por análises de FTIR, UV, RMN, DSC, análise elementar e TGA. Uma maior massa molecular, maior rendimento e uma melhor conversão do grupo azida a GAP foram obtidos com a adição de epicloridrina sobre a azida de sódio e usando DMF como solvente.
\end{abstract}

Palavras-chave: Síntese, caracterização do GAP, materiais energéticos.

\section{Glycidyl Azide Polymer (GAP). I. Syntheses and Characterization}

Abstract: GAP is an aliphatic polyether that includes hydroxyl groups and highly energetic azide groups. Thus, it is an energetic material that can be used as binder or plasticizing agent in propellants and explosive mixtures. The glycidyl azide polymer (GAP) was synthesized and characterized by direct conversion of epichlorohydrin. GAP was synthesized by reaction of sodium azide, epichlorohydrin, and some extensor alcohols. The investigation focused on the effects of some key reaction parameters including reagent proportions, reaction time and two different solvents. The product was characterized by FTIR, UV, NMR, DSC, elemental analysis, TGA and GPC. The species were also evaluated through molecular weight (GPC), glass transition temperature (DSC), ignition time and sensitivity.

Keywords: Syntheses, GAP characterization, energetic compound.

\section{Introdução}

A primeira referência histórica de uso de materiais energéticos vem de 300 anos a.C., quando a pólvora negra foi utilizada pelos Chineses em cerimoniais. A aplicação destes materiais foi intensificada no início do século XIX, com a nitração de compostos para produzir explosivos de alta energia. Desde então, novos materiais energéticos surgiram, de certa forma acompanhando a evolução da ciência dos materiais.

Os materiais poliméricos foram introduzidos nas composições de explosivos para melhoria de algumas propriedades como manuseio, transporte e estocagem ${ }^{[1]}$. Primeiro, a matriz polimérica pode servir como receptora de grupos energéticos chamados de explosóforos, que contribuem com energia adicional ao material ${ }^{[2]}$. Posteriormente, a matriz teve incluída partículas energéticas em sua própria estrutura. Foram criados então os ligantes energéticos - binders $^{[3,4]}$, que agem como aglomerantes e ainda podem fazer ligações cruzadas com explosóforos ${ }^{[5]}$. O poli (metil azoteto de glicidila) GAP é um exemplo de aglomerante, que contém grupamentos azidas em sua cadeia (Figura 1), responsáveis pelo aumento do conteúdo energético da composição ${ }^{[6]}$.

Neste trabalho foi realizada a síntese e a caracterização do poli (metil azoteto de glicidila) de baixa massa molecular através da conversão direta da epicloridrina a GAP, para ser usado como plastificante energético em composições explosivas. A análise do produto foi realizada considerando a massa molecular numérica média como objetivo e variando os seguintes parâmetros: os álcoois extensores, os tempos de reações, solventes, a metodologia de produção e mostrando possível rota sintética do material. A caracterização dos compostos obtidos foi feita através de espectrometria no infravermelho (FTIR), ultravioleta (UV), ressonância magnética nuclear (RMN), análise elementar (CHN), GPC (cromatografia por permeação em gel), TGA (análise térmica gravimétrica) e DSC (Calorimetria diferencial de varredura).

\section{Fundamentos Teóricos}

A síntese do GAP foi citada por Vandenberg em 1972, tendo como ponto de partida a poli (epicloridrina) amorfa ou cristalina com peso molecular de 25.000 g. mol $^{-1[7]}$. Entretanto, apenas em 1982 numa conferência do ICT, o GAP teve a sua utilidade associada à fabricação de propelentes anunciada pela primeira $\mathrm{vez}^{[8]}$.

As primeiras sínteses consistiam apenas da substituição do cloro pelo grupo azida, geravam um produto linear com um peso molecular de até 3.000 g. $\mathrm{mol}^{-1}$ e funcionalidade $2^{[9]}$. Depois, um polímero linear atático com grupos hidroxílicos secundários e peso molecular variando entre 1000 e 3000 g.mol ${ }^{-1}$ foi obtido ${ }^{[8]}$. Alguns anos mais tarde, surgiram o GAP ramificado e com funcionalidade variando entre 6,9 e $11,0^{[9]}$. Este processo envolve a degradação da poli (epicloridrina) comercial, seguida pela sua reação com azida de sódio num solvente apropriado ${ }^{[9]}$. Um GAP linear, com funcionalidade maior que 2 , foi obtido pela epoxidação 
da PECH, com peso molecular médio $\left(\mathrm{M}_{\mathrm{n}}\right)$ de 2.000 g.mol ${ }^{-1}$ em condições básicas ou ácidas seguida pela abertura do anel formado e azidação ${ }^{[10]}$.

O GAP com baixo peso molecular pode ser utilizado como plastificante energético. É produzido através de um só processo pela direta conversão de epicloridrina em $\mathrm{GAP}^{[11]}$. A reação resumida é apresentada na Figura 2.

\section{Experimental}

\section{Reagentes}

Os reagentes usados foram os seguintes: epicloridrina marca Fluka, grau de pureza 98\%, diclorometano marca Aldrich, grau de pureza 99\% butanodiol 1,4, marca Aldrich, grau de pureza 99\%, etanodiol, marca Aldrich, grau de pureza 99\%, glicerol, marca Aldrich, grau de pureza 99\%, dietilenoglicol, marca Carlo Erba, grau de pureza 99\%; dimetilformamida (DMF) e dimetilsulfóxido (DMSO) marca Merck, grau de pureza 99,5\% e azida de sódio marca Vetec, grau de pureza $99 \%$.

A epicloridrina e o diclorometano foram tratados com sulfato de cálcio e posteriormente submetidos à destilação para remoção de traços de umidade. Todos os outros reagentes foram utilizados como recebidos.

\section{Equipamentos}

As reações foram realizadas em balão de vidro com três bocas com condensador para recuperação dos reagentes voláteis. Foram também usados termômetros e placa de aquecimento com agitador magnético. Todos os procedimentos de segurança nas manipulações dos reagentes tóxicos e explosivos foram severamente observados.

Os espectros de infravermelho dos compostos foram obtidos em um Espectrofotômetro de Infravermelho com Transformada de Fourier (FTIR), Perkin Elmer, modelo 1710. As amostras sendo líquidas, foram aplicadas diretamente sobre células de $\mathrm{NaCl}$. Os

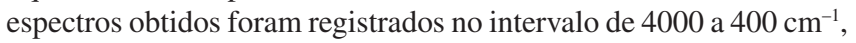
tendo resolução de $4 \mathrm{~cm}^{-1}$ e ganho de 1,40 varreduras.

Os espectros de $\mathrm{RMN}-{ }^{1} \mathrm{H}$ e $\mathrm{RMN}-{ }^{13} \mathrm{C}$ das substâncias foram obtidos em um espectrômetro de Ressonância Magnética Nuclear - RMN (Varian, modelo Unity-300), com freqüência

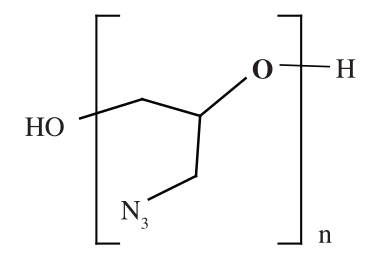

Figura 1. Molécula do GAP.

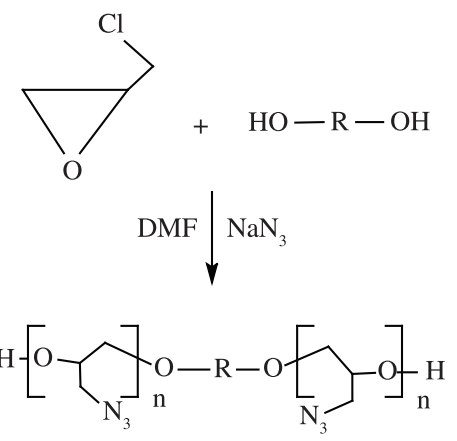

Figura 2. Reação do GAP com baixa massa molecular. de $300 \mathrm{MHz}$ para ${ }^{1} \mathrm{H}$ e $75 \mathrm{MHz}$ para ${ }^{13} \mathrm{C}$. Cerca de $50 \mathrm{mg}$ do produto purificado, previamente secos sob luz infravermelha, foi solubilizado em cerca de $0,7 \mathrm{~mL}$ de solventes deuterados. Os sinais de ressonância foram registrados em uma escala em ppm relativos ao padrão interno tetrametilsilano (TMS).

A análise térmica gravimétrica (TGA) foi obtida em aparelho TGA-50 modelo TA 2100. A temperatura de decomposição térmica do material foi considerada como igual à temperatura de ignição. A análise foi feita em atmosfera inerte, com cadinho de platina e massa de material de aproximadamente $10 \mathrm{mg}$. A análise iniciou a $20^{\circ} \mathrm{C}$ e foi levada a $500{ }^{\circ} \mathrm{C}$ com razão de aquecimento de $10{ }^{\circ} \mathrm{C} / \mathrm{min}$.

$\mathrm{O}$ espectrômetro de ultravioleta foi obtido em um Shimadzu 160 A, usando uma solução muito diluída em solvente tetrahidrofurano (THF) nas faixas de concentração de $0,0010(\mathrm{p} / \mathrm{v})$. A ressonância do grupo azida foi detectada em um comprimento de onda de $236 \mathrm{~nm}$.

Os polímeros foram caracterizados em um cromatógrafo de exclusão de tamanhos, modelo Waters 515 HPLC Pump, com um Detector - Waters 2414 Índice de Refração e Trio de colunas da Waters Ultrastyragel Linear, 500 Å. A fase móvel utilizada foi THF a uma vazão de $1,00 \mathrm{~mL} / \mathrm{min}$ e a temperatura de $30{ }^{\circ} \mathrm{C}$.

A análise de calorimetria exploratória diferencial foi conduzida sob atmosfera de nitrogênio a partir do aquecimento de $-100^{\circ} \mathrm{C}$ até a temperatura de $50{ }^{\circ} \mathrm{C}$, a uma taxa de aquecimento de $10{ }^{\circ} \mathrm{C} / \mathrm{min}$.

A análise das porcentagens de carbono, hidrogênio e oxigênio foi feita em um aparelho Instruments EA1110 CHN-O CE, com fluxo de oxigênio a $925{ }^{\circ} \mathrm{C}$. Os produtos colocados para queima no aparelho foram analisados em testes de duas corridas para cada reação.

\section{Rotas para a síntese do GAP}

A síntese do GAP seguiu a metodologia proposta por Ahad ${ }^{[1]}$. A epicloridrina e o etilenoglicol foram dissolvidos em DMF sob atmosfera inerte $\left(\mathrm{N}_{2}\right)$. A mistura foi pré-aquecida a $75{ }^{\circ} \mathrm{C}$ e a agitação iniciada.

A azida de sódio foi então lentamente adicionada ao meio. Nesta etapa, devido à reação exotérmica da abertura do anel epóxi da epicloridrina, deve-se controlar a temperatura de forma que a mesma não atinja $85^{\circ} \mathrm{C}$. Quando a adição da azida não promover mais alteração na temperatura, a polimerização foi considerada terminada. Após este período, foi adicionado cloreto de lítio anidro. O cloreto de lítio ou sais de amônio quaternário atuam como catalisadores, pois aumentam a solubilidade da azida no solvente e, consequentemente, a sua nucleoficidade. Após o período de adição, a temperatura foi elevada a $90{ }^{\circ} \mathrm{C}$ e mantida a agitação por 20 horas.

$\mathrm{O}$ produto de todas as corridas sofreu o mesmo processo de lavagem e purificação. Primeiro, o produto foi lavado com água quente $\left(90^{\circ}\right)$ para retirar os reagentes. Depois, o produto da reação foi dissolvido em cloreto de metileno, de forma que esta solução orgânica fosse lavada a frio cinco vezes com água. Finalmente, a solução foi seca sobre sulfato de magnésio anidro e coluna de silicagel.

O solvente foi então retirado utilizando-se rota evaporador sob vácuo $(0,15$ Torr $)$ e $40{ }^{\circ} \mathrm{C}$ por cerca de uma hora, sendo essa etapa em termos de segurança, a mais perigosa devido à alta reatividade da azida com o cloreto de metileno. O produto obtido apresentou coloração amarelo âmbar.

\section{Planejamento de experimentos}

As variáveis avaliadas foram o tempo de reação (15 e 20 minutos), o tipo de álcool extensor (etanodiol, dietilenoglicol e 1,4 butanodiol) e a proporção de álcool/monômero. Também foram feitas avaliações utilizando dois solventes diferentes, DMF e DMSO. 


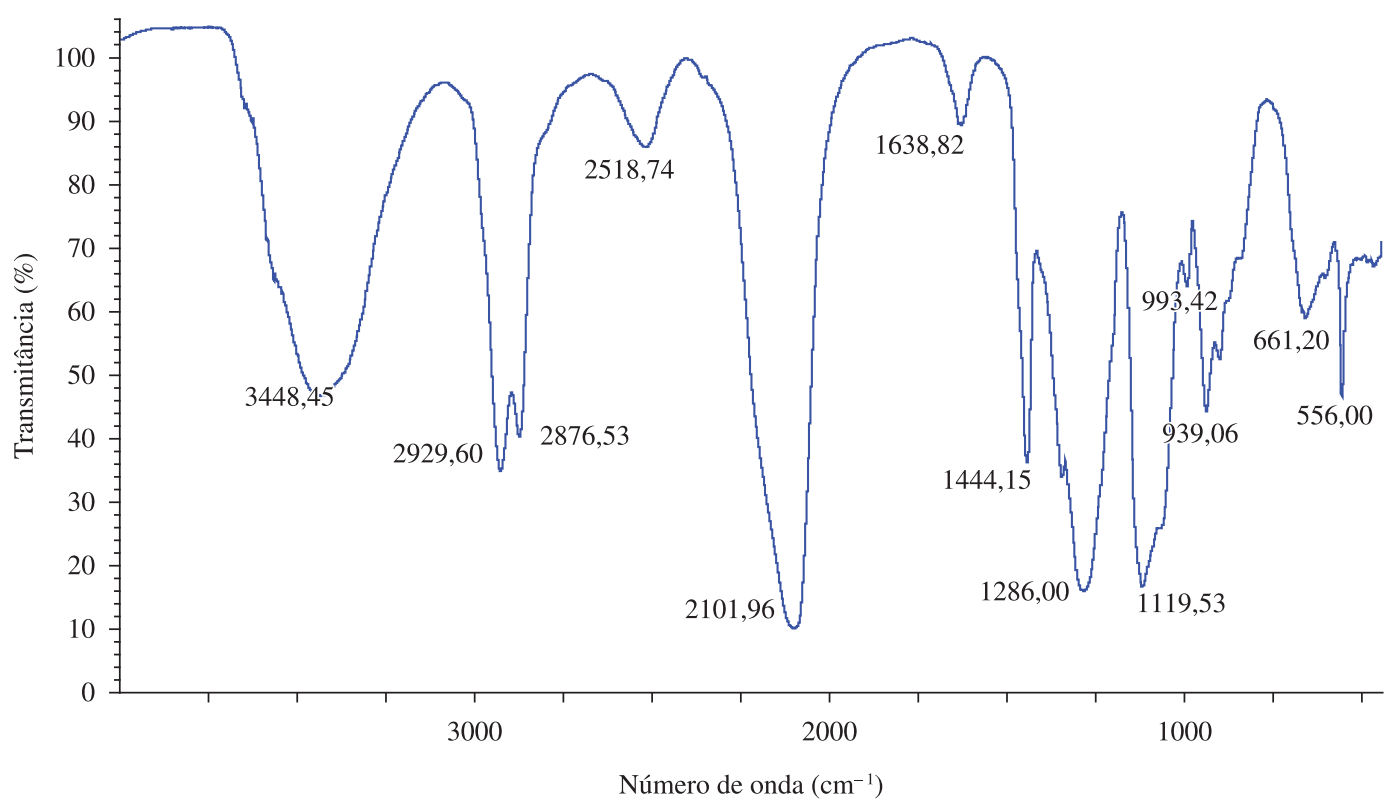

Figura 3. Espectro de IV do GAP.

A ordem de adição dos reagentes também foi avaliada. Ao invés de adicionar a azida de sódio, conforme ocorre no método anterior $^{[11]}$, a epicloridrina foi adicionada ao meio. Portanto, $30 \mathrm{~g}$ de azida foram solubilizadas em um balão de três bocas, junto com $60 \mathrm{~g}$ de solvente, $3 \mathrm{~g}$ do álcool que funciona como extensor de cadeia $\mathrm{e}$ $3 \mathrm{~g}$ de cloreto de lítio. As temperaturas das reações foram mantidas por $90{ }^{\circ} \mathrm{C}$ por até vinte horas, seguindo todos os procedimentos e proporções de reagentes semelhantes à metodologia anterior ${ }^{[11]}$.

\section{Resultados e Discussão}

A caracterização do GAP foi feita por meio das técnicas de análise IV, UV, CHN, RMN e DSC. A caracterização foi feita para o produto de todas as reações ${ }^{[12]}$, mas, somente parte delas está apresentada aqui.

A espectrometria de infravermelho do GAP, ver Figura 3, apresenta para o grupo azida $\left(\mathrm{C}-\mathrm{N}_{3}\right)$ uma absorção intensa em $2100 \mathrm{~cm}^{-1}$; para o grupo éter uma absorção próxima a $1118-1119 \mathrm{~cm}^{-1}$, para hidroxilas terminais uma absorção larga em aproximadamente $3550 \mathrm{~cm}^{-1}$ e ausência da ligação $(\mathrm{C}-\mathrm{Cl})$ para absorção em torno de $744 \mathrm{~cm}^{-1[3,13]}$.

Na espectrometria de Ultravioleta foi detectada a absorção da ressonância relacionada ao grupo azida na cadeia do material sintetizado em torno de $236 \mathrm{~nm}^{[14,15]}$. A análise elementar de CHN confirmou uma porcentagem de nitrogênio resultado da substituição do grupo cloro da epicloridrina pelo nitrogênio correspondente ao grupamento azida nas amostras do GAP.

Outra análise utilizada nesse trabalho para caracterizar a estrutura do GAP foi a de RMN de ${ }^{13} \mathrm{C}$ e de ${ }^{1} \mathrm{H}$ (Figuras 4-7). Devido ao acoplamento dos hidrogênios e carbonos no polímero, várias absorções foram encobertas, dificultando as análises. Contudo, foram obtidos resultados similares para os diversos produtos mesmo variando os álcoois, tempos de reação, proporção dos reagentes e metodologias ${ }^{[12]}$. Os valores para as absorções de $\mathrm{RMN}$ de ${ }^{1} \mathrm{H}$ e de RMN de ${ }^{13} \mathrm{C}$ encontradas nos compostos sintetizados aqui foram numerados para melhor visualização (Figuras 5 e 7).

O RMN de ${ }^{1} \mathrm{H}$ apresenta absorções de $\mathrm{CH}_{2}$ (1) e $\mathrm{CH}$ (2) ligados a oxigênio e tem valores entre 3,5 e 3,8 ppm e $\mathrm{CH}_{2}$ (3), ligado ao grupo azida em 3,4 ppm. OH (4) de hidroxila em 2,57 ppm ${ }^{[14,16]}$.

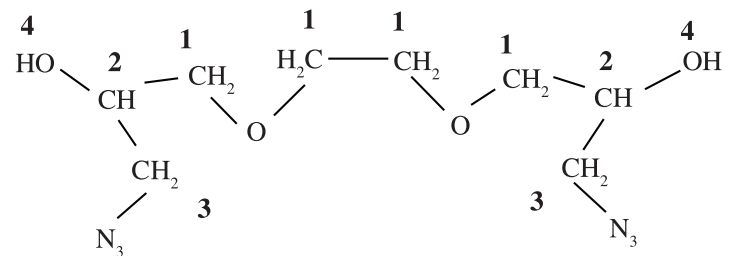

Figura 4. Estrutura GAP RMN ${ }^{1} \mathrm{H}$.

Tabela 1. Rendimentos de reação e peso molecular do GAP.

\begin{tabular}{ccccc}
\hline Álcool & $\begin{array}{c}\text { Álcool/ } \\
\text { Monômero }\end{array}$ & $\begin{array}{c}\text { Tempo } \\
\text { (horas) }\end{array}$ & Rendimentos & $\begin{array}{c}\text { Mn } \\
\left(\text { g.mol }^{-1}\right)\end{array}$ \\
\hline $\begin{array}{c}\text { Etanodiol } \\
\text { Dietileno } \\
\text { glicol }\end{array}$ & $0,1 / 1$ & 20 & $76 \%$ & 262 \\
$\begin{array}{c}\text { Dietileno } \\
\text { glicol }\end{array}$ & $0,1 / 1$ & 20 & $77 \%$ & 394 \\
$\begin{array}{c}\text { Butanodiol } \\
\text { Butanodiol }\end{array}$ & $0,1 / 1$ & 15 & $78 \%$ & 270 \\
Butanodiol & $0,1 / 1,5$ & 15 & $76 \%$ & 205 \\
\hline
\end{tabular}

As absorções analisadas no RMN de ${ }^{13} \mathrm{C}$ são mostradas nas Figuras 6 e 7. O valor para a absorção $\mathrm{CH}_{2}$ (4) ligado ao grupo azida é mostrado em $52 \mathrm{ppm}$; $\mathrm{CH}_{2}$ (2) do monômero ligado ao álcool extensor em 72 ppm; $\mathrm{CH}_{2}$ (1) dos álcoois em 69-70 ppm; $\mathrm{CH}$ (3) em 77-79 ppm $^{[14,16]}$.

A análise de TGA indica a temperatura de decomposição térmica do material. Aqui, a taxa de aquecimento foi de $10^{\circ} \mathrm{C} / \mathrm{min}$ em atmosfera de nitrogênio. Considera-se que a perda de massa iniciou em $200{ }^{\circ} \mathrm{C}$ e terminou em $224{ }^{\circ} \mathrm{C}$ (Figura 8). A temperatura de ignição do GAP pode ser considerada como igual à temperatura inicial da perda de massa ${ }^{[13,17]}$, ou seja, $200{ }^{\circ} \mathrm{C}$.

As reações da Tabela 1 foram feitas segundo a metodologia da Patente ${ }^{[11]}$ com adição de azida de sódio. Os resultados das primeiras análises obtidos através do GPC, com as variações feitas nas proporções dos reagentes, tempos de reações e álcoois extensores, mostram diferenças nas massas moleculares e nos rendimentos.

Foi observado que há um acréscimo da massa molecular com o aumento do tempo da reação. A reação é aniônica por abertura de 

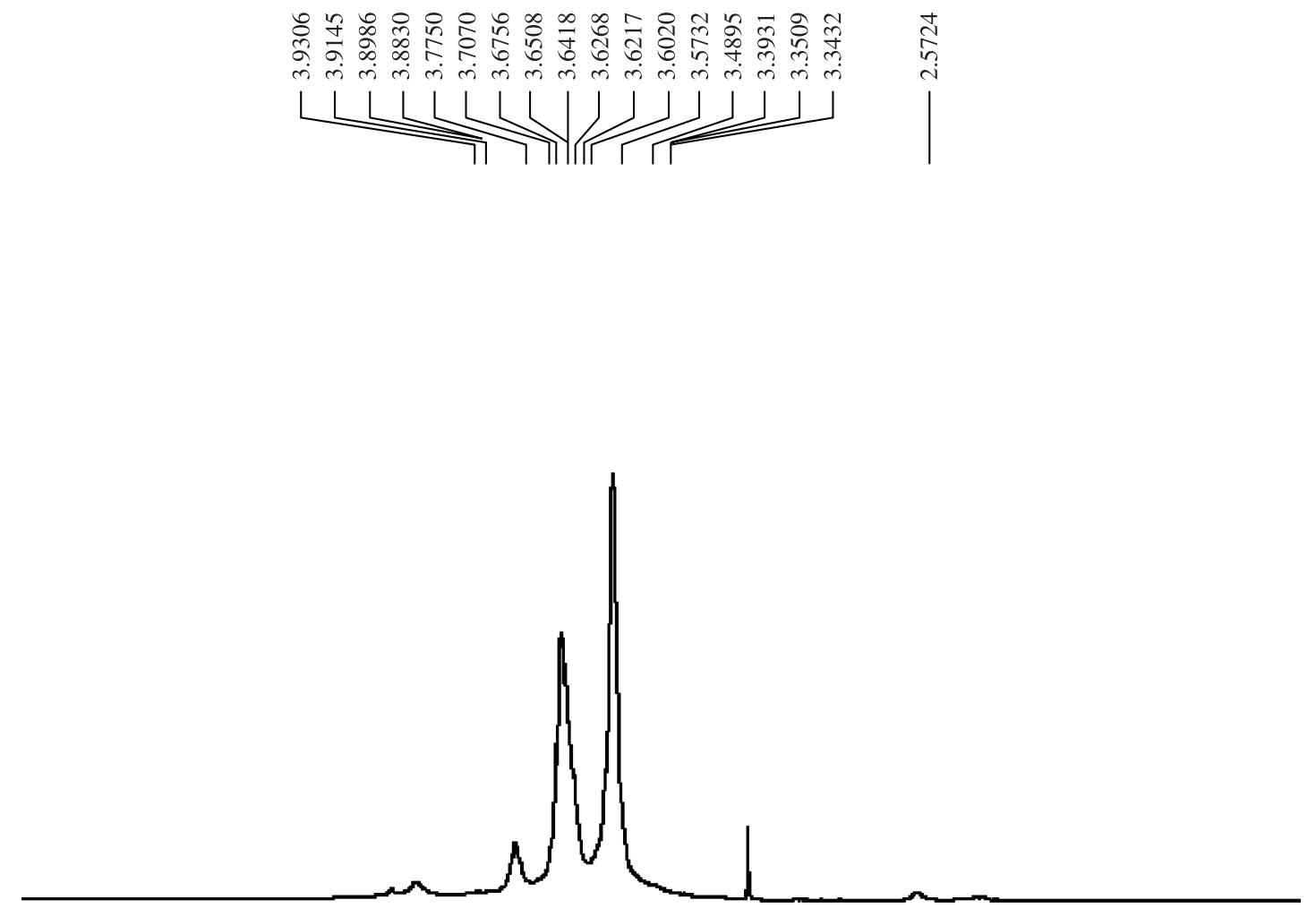

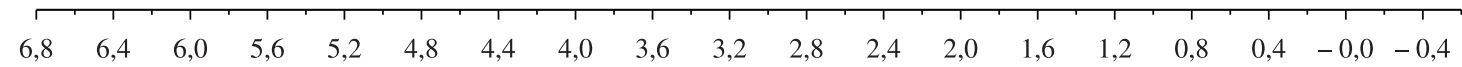

Figura 5. RMN ${ }^{1} \mathrm{H}$ GAP etanodiol ${ }^{[14]}$.

anel epóxido, seguida por substituição do cloro pela azida de sódio. Nessa análise, pode ter ocorrido a presença de espécies reativas no meio reacional, que com o passar do tempo reagiram, levando a um aumento na massa molecular do GAP.

O tempo é significativo na obtenção de uma maior massa molecular na síntese do GAP. O grande problema no uso de um tempo maior que o descrito pela Patente ${ }^{[11]}$, está na periculosidade da reação, que é explosiva. Nesse trabalho a reação foi exposta a um tempo maior de síntese, em torno de 24 horas. Nessa variação de tempo foi observado intenso desenvolvimento de vapor. Ao perceber isto, a corrida foi interrompida com a paralisação da agitação e do aquecimento. Por questões de segurança, o tempo da reação foi estipulado como no máximo de 20 horas.

Quando a variação foi feita nas proporções dos reagentes, foi observado que a diminuição na proporção do álcool extensor utilizado levou a redução da massa molecular do GAP. O aumento na proporção do monômero provoca um decréscimo na massa molecular. Isso é devido a uma maior quantidade de monômero ECH no meio reacional, o que provoca uma maior formação de compostos cíclicos que reduzem a massa molecular numérica média do GAP ${ }^{[18]}$.

A síntese da PECH pode apresentar picos de baixa massa molecular no GPC, o que indica presença de cíclicos ${ }^{[19]}$. Neste trabalho, observou-se este comportamento para a síntese do GAP a partir de 1,4-butanodiol com excesso de $\mathrm{ECH}$, como pode ser visto na Figura 9.

Nas reações feitas com a nova ordem de adição de reagentes, com dois tipos de solventes e com os diferentes extensores, foram

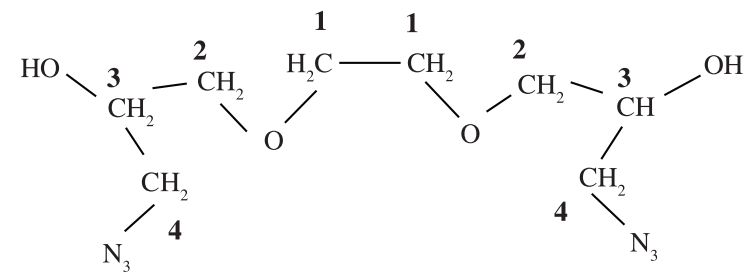

Figura 6. Estrutura GAP RMN ${ }^{13} \mathrm{C}$.

Tabela 2. Rendimento do GAP - diferentes solventes e nova ordem de adição.

\begin{tabular}{cccc}
\hline Etanodiol & Dietilenoglicol & Butanodiol & Glicerol \\
\hline $\mathrm{DMF}=79 \%$ & $\mathrm{DMF}=80 \%$ & $\mathrm{DMF}=80 \%$ & $\mathrm{DMF}=82 \%$ \\
$\mathrm{DMSO}=$ & $\mathrm{DMSO}=79 \%$ & $\mathrm{DMSO}=79 \%$ & $\mathrm{DMSO}=81 \%$ \\
$78 \%$ & & & \\
\hline
\end{tabular}

observados maiores níveis de rendimento (Tabela 2), em relação aos obtidos com a metodologia origina ${ }^{[11]}$.

Nas sínteses realizadas com diferentes tipos de álcoois, dois tipos de solventes e dois métodos diferentes de reação, é possível observar os valores das massas através do GPC. De acordo com a Tabela 3, a massa molecular do GAP apresenta variações para os dois tipos de solventes e para os dois métodos de síntese. Com o uso da metodologia da patente ${ }^{[11]}$ nas sínteses, houve um decréscimo na massa molecular em comparação à metodologia nova de adição da epicloridrina. Isso pode ser atribuído ao excesso de monômeros epicloridrina no meio reacional, proporcionando um aumento na formação de compostos cíclicos de baixa massa molecular ${ }^{[18]}$. Quando a síntese ocorre pelo método novo da adição lenta de 

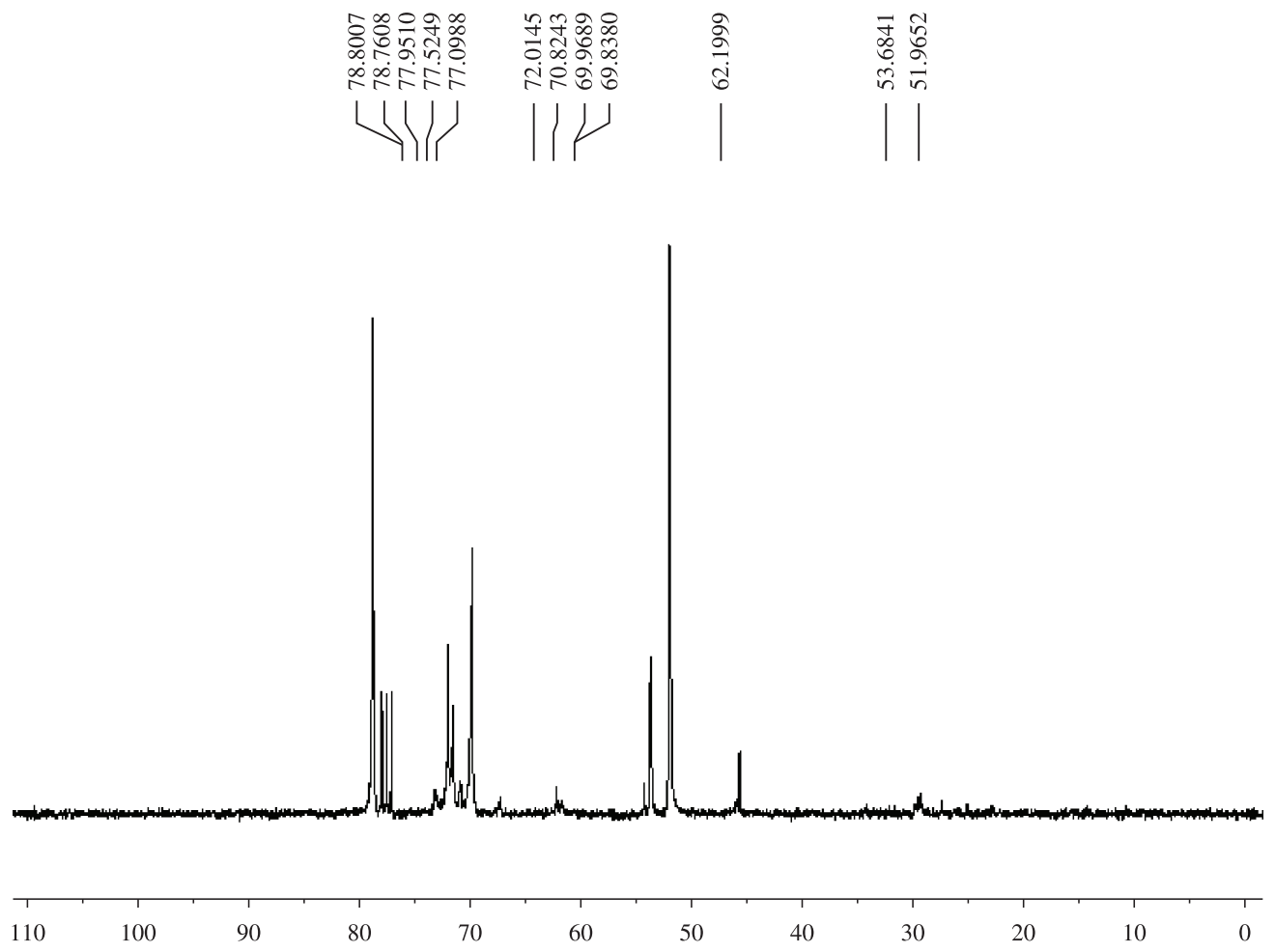

Figura 7. RMN ${ }^{13} \mathrm{C}$ GAP etanodiol.

ECH, o meio reacional fica limitado, com uma quantidade mínima de monômero epicloridrina. Isso dificulta a formação dos ciclos aumentando a massa molecular média do GAP ${ }^{[12,18]}$.

Nas variações analisadas através da utilização da DMF e do DMSO, foi observado que o uso do solvente DMF aumenta os valores das massas moleculares assim como o rendimento das reações. Isso pode estar relacionado com vários fatores, um deles é a interação entre o monômero e o solvente, quanto maior essa interação, maior a conversão de ECH em GAP ${ }^{[18]}$.

A substituição do grupo cloro para grupo azida pode ser confirmada por análise elementar CHN. Foram analisados os compostos preparados com a nova metodologia e com a metodologia da Patente ${ }^{[11]}$, ambos com a utilização dos dois solventes e com o mesmo tipo de álcool. Os resultados estão apresentados na Tabela 4. O valor obtido na utilização do solvente DMF com o procedimento de síntese da patente é de 39,8\% de nitrogênio na amostra. Com o mesmo procedimento e com o solvente DMSO, a taxa de conversão de nitrogênio foi um pouco menor $(39,2 \%)$. Quando a síntese foi feita com a nova metodologia e com o uso da DMF como solvente, ocorreu um aumento na porcentagem de nitrogênio no material de $1,7 \%$ em relação à metodologia de síntese original da patente utilizando o mesmo solvente. Esse aumento indica um ganho de energia para o material, já que cada grupamento azida é responsável por $80 \mathrm{kcal}^{\mathrm{mol}}{ }^{-1}$ e uma elevada entalpia de formação conforme já mencionado $^{[20]}$.

Foi constatado nessas variações através das análises do CHN que a metodologia nova de adição da ECH é mais eficaz. Nessa metodologia ocorre um aumento na velocidade da substituição de cloro por azida nos dois tipos de solventes utilizados. Conforme mencionado, o novo método diminui a formação de compostos cíclicos e aumenta a velocidade de substituição do nitrogênio, relacionado ao grupo azida.

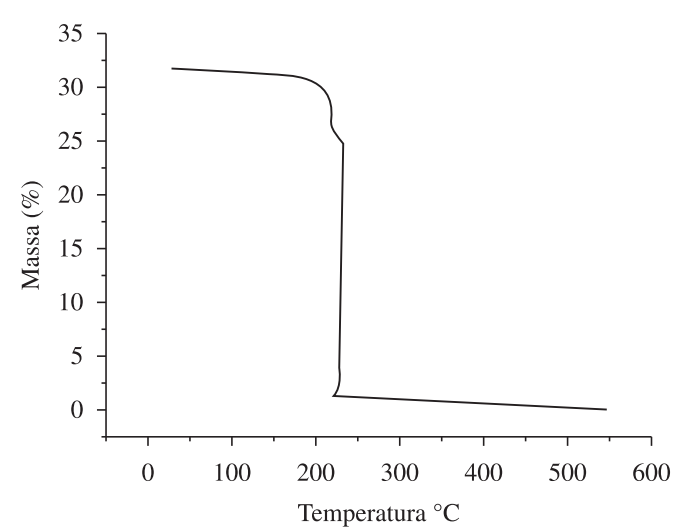

Figura 8. TGA do GAP etanodiol ${ }^{[13]}$.

Tabela 3. Massas moleculares do GAP para diversas condições.

\begin{tabular}{ccccc}
\hline $\begin{array}{c}\text { Álcool } \\
\text { extensor }\end{array}$ & $\begin{array}{c}\text { Método de } \\
\text { adição }\end{array}$ & Solvente & $\begin{array}{c}\text { Tempo } \\
\text { (horas) }\end{array}$ & $\begin{array}{c}\text { MM } \\
\left(\text { g.mol }^{-1} \text { ) }\right.\end{array}$ \\
\hline 1,4 Butanodiol & Adição ECH & DMF & 20 & 366 \\
1,4 Butanodiol & Adição ECH & DMSO & 20 & 340 \\
1,4 Butanodiol & Adição azida & DMF & 20 & 324 \\
1,4 Butanodiol & Adição azida & DMSO & 20 & 310 \\
Dietileno & Adição azida & DMF & 20 & 394 \\
Glicol & Adição ECH & DMF & 20 & 495 \\
Glicerol & Adiç̃̃o ECH & DMSO & 20 & 431 \\
Glicerol & & & &
\end{tabular}

Tabela 4. Análise elementar por $\mathrm{CHN}$.

\begin{tabular}{ccccc}
\hline Álcool & $\% \mathbf{~ N}$ & $\% \mathbf{C}$ & Solventes & Metodologia \\
\hline Butanodiol & 39,8 & 35,0 & DMF & Patente \\
Butanodiol & 39,2 & 36,3 & DMSO & Patente \\
Butanodiol & 41,5 & 35,0 & DMF & Novo método \\
Butanodiol & 41,3 & 35,1 & DMSO & Novo método
\end{tabular}




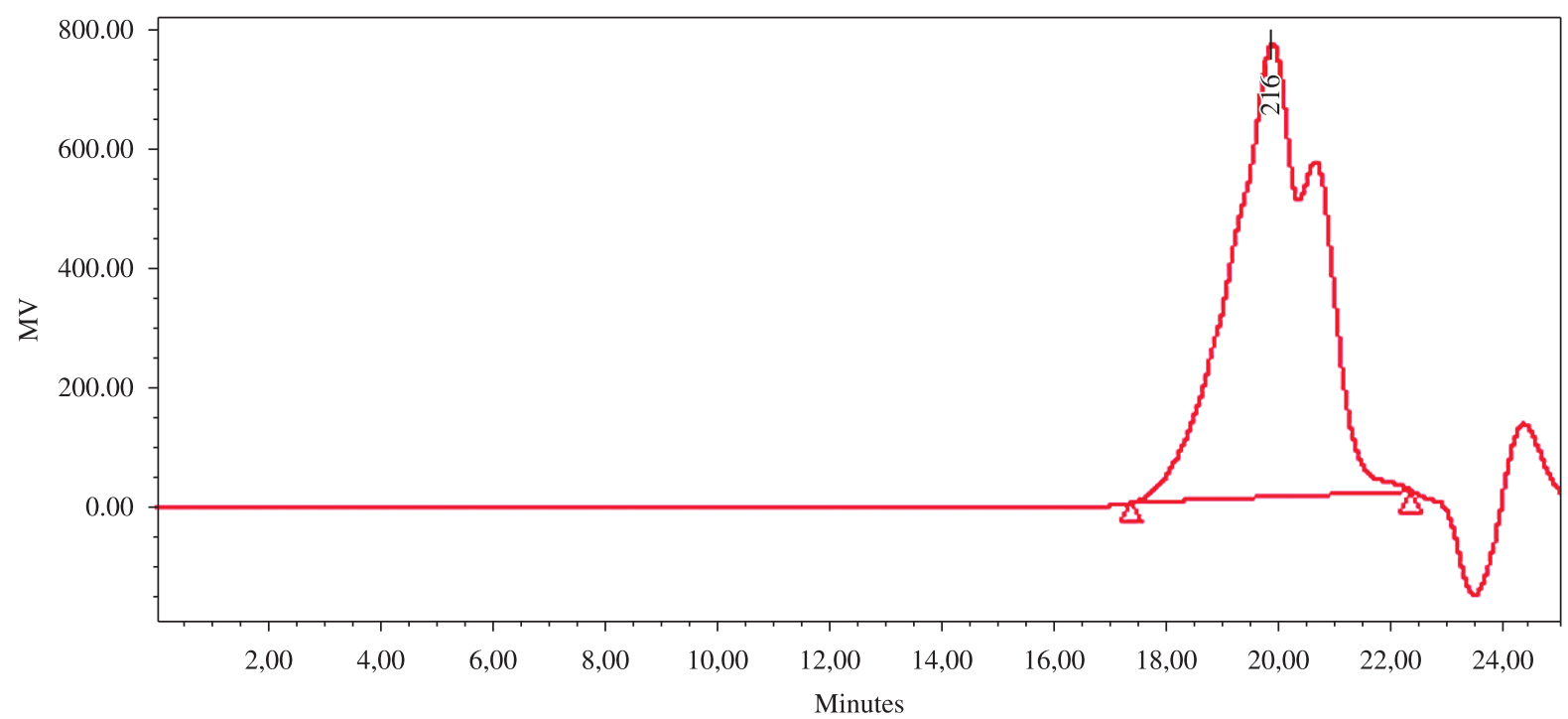

Figura 9. GPC GAP de menor massa molecular.

Tabela 5. Análise do DSC ( $\mathrm{T}_{\mathrm{g}}$ ) temperatura de transição vítrea do GAP.

\begin{tabular}{ccc}
\hline $\begin{array}{c}\text { Álcoois } \\
\text { extensores }\end{array}$ & $\mathrm{MM}\left(\mathrm{g} \cdot \mathrm{mol}^{-1}\right)$ & Transição vítrea $\left({ }^{\circ} \mathrm{C}\right)$ \\
\hline Etileno glicol & 262 & $-65,76$ \\
Butanodiol & 366 & $-69,81$ \\
Dietileno glicol & 394 & $-71,67$ \\
Glicerol & 495 & $-74,38$
\end{tabular}

Com a utilização do solvente DMF no novo método de adição da $\mathrm{ECH}$, ocorre um aumento na velocidade de substituição do cloro pelo grupo azida.

As interações do polímero-solvente e do monômero-solvente são fatores determinantes na formação dos compostos cíclicos ${ }^{[21]}$. A formação de ciclos causa uma interação polímero-polímero mais intenso do que a do polímero-solvente ${ }^{[22]}$, levando a um baixo rendimento da reação e a uma menor massa molecular média ${ }^{[23]}$.

Os aumentos observados no rendimento das reações de síntese do GAP, no aumento das massas moleculares e nas taxas de conversões, com o uso da DMF, confirmam a maior interação desse solvente com os reagentes. Foi observado também que com a utilização do novo método ocorre uma baixa formação de compostos cíclicos. A menor quantidade dos compostos cíclicos influencia nos aumentos dos valores das massas e dos rendimentos das reações do GAP.

A temperatura de transição vítrea $(\mathrm{Tg})$ foi obtida por DSC para cada um dos polímeros com os diferentes álcoois extensores. Os resultados estão na Tabela 5 .

As massas obtidas com os diferentes álcoois extensores indicam que os maiores números de ramificações e conformações espaciais dos materiais, abaixam a temperatura de transição vítrea, $\mathrm{Tg}^{[24]}$. Os maiores números de ramificações e conformações dificultam as interações entre as moléculas, impedindo que elas deslizem mais umas nas outras, o que leva a uma temperatura de $\mathrm{Tg}$ menor ${ }^{[25]}$.

Os baixos valores da temperatura de transição vítrea é uma característica do $\mathrm{GAP}^{[26]}$. O comportamento mecânico de um propelente durante os períodos de tensão está relacionado com suas propriedades a diferentes temperaturas. As temperaturas encontradas aqui nesse trabalho se encontram dentro de uma faixa boa para uso em propelentes ${ }^{[26]}$.

\section{Conclusão}

Neste trabalho foi obtido em laboratório o Poli (metil azoteto de glicidila) (GAP) com baixo peso molecular através da conversão direta da epicloridrina, rota originalmente proposta na patente US 4, 871, 438. Através das técnicas tradicionais de análise foi possível caracterizar e confirmar o material e algumas de suas propriedades relacionadas ao seu uso como plastificante energético em propelentes.

As massas moleculares obtidas através do GPC variaram com o uso dos diferentes álcoois extensores, variados tempos de reações, solventes, proporções de reagentes e da ordem de adição dos reagentes. Uma maior massa molecular foi obtida com DMF como solvente e com a adição de epicloridrina, proposto neste trabalho. Esse método novo com o solvente DMF foi mais eficaz na obtenção de uma maior massa molecular, maior rendimento e uma melhor conversão do grupo azida no material.

\section{Agradecimentos}

Os autores agradecem ao Instituto de Macromoléculas Eloisa Mano (IMA-UFRJ) na pessoa da Professora Bluma Guenther Soares, pela realização das análises de GPC.

\section{Referências Bibliográficas}

1. Kishore, K. \& Sridhara, K. - "Solid Propellant Chemistry", Desidoc, Defense R\&D Organization, New Delhi (1998).

2. Arisawa, H. \& Brill, T. B. - Combust. Flame, 112, 4, p.533 (1998). http://dx.doi.org/10.1016/S0010-2180(97)00162-4

3. Provatas, A. - "Energetic Polymers and Plasticizers For Explosive Formulation -A Review of Recent Advances", DSTO-Tr-0966, DSTO, Australia (2000).

4. Frankel, L. R.; Grant, J. E. \& Flanagan, J. E. - J. Propul. Power, 18, p.560 (1992).

5. Klager, K. - "Polyurethanes, The Most Versatile Binder For Solid 'Composites' Propellants", in: Aiaa/Sae/Asme 20th Joint Propulsion Conference, Cincinnati - Ohio (1984).

6. Çöçmez, A.; Erisken, C.; Yilmazer, Ü.; Pekel, F. \& Özkar, S. - J. Appl. Polym Sci., 67, p.1457 (1998). http://dx.doi.org/10.1002/(SICI)10974628(19980222)67:8<1457::AID-APP11>3.0.CO;2-Z 
7. Ping, W.; Zhongjun, X.; Zhong, W. \& Changging, L. - "Investigation Of High Molecular Weight Gap", in: The 27th International Annual Conference of ICT, Karlsruhe - Germany, p. 25 (1996).

8. Chen, B.; Yan, H.; Jia, H.; Li, J. \& Dong, S. - J. Propul. Power, 11, 4, p.838 (1995).

9. Ahad, E. - "Branched Hydroxy Terminated Azido Polymers", in: The 21th International Annual Conference of ICT, p.5, Karlsruhe - Germany (1991).

10. Ampleman, G.; Désilets, S. \& Marois, A. - "Energetic Thermoplastic Elastomers Based On Glycidyl Azide Polymers With Increased Functionality", in: The 27th International Annual Conference of ICT, p.132, Karlsruhe - Germany (1996).

11. Ahad, E. "Direct Conversion of Epichlorohydrin to Glycidyl Azide Polymer", US Patent 4,891,438 (1990).

12. Ribeiro, S. P. - "Síntese e Caracterização do Poli(metilazoteto de Glicidila) GAP", Dissertação de Mestrado, Instituto Militar de Engenharia, Brasil (2010).

13. Sciamareli, J.; Costa, J. R.; Takahashi, M. F. K.; Diniz, M. F.; Lourenço, V. L.; David, L. H., Iha, K.; Miyano, M. H. \& Ferreira, C. - Polímeros, 19, 2, p.117 ( 2009). http://dx.doi.org/10.1590/S010414282009000200008

14. Silverstein, R. M.; Bassler, G. C. \& Morril, T. C. - "Identificação Espectrométrica de Compostos Orgânicos”, 5.ed., Guanabara Koogan, Rio de Janeiro (2000).

15. Eroglu, M. S.; Hazer, B. \& Guven, O. - Polym. Bull., 36, 6, p.695, (1996).

16. Desilets, S. \& Villeneuve, S. - "Structural characterization of glycidyl azide polymer binders by NMR spectroscopy", in: The 25th International Annual Conference of ICT, p. 96-1, Karlsruhe - Germany (1994).
17. Korobeinichev, O. P.; Kuibida, L. V.; Volkov, E. N. \& Shmakov, A. G. - Combust. Flame, 129, p.136 (2002). http://dx.doi.org/10.1016/ S0010-2180(01)00370-4

18. Kubisa, K. - Prog. Polym. Sci., 24, p.1409 (1999). http://dx.doi. org/10.1016/S0079-6700(99)00028-3

19. Yu, S. H. - Polym. prepr., 25, 1, p.117 (1984).

20. Kawamoto, A. M. et al. - "Synthesis and Characterization of Energetic Aba-Type Thermoplastics Elastormers for Propellants Formulations", in: The 37th International Annual Conference of ICT, Karlshure, Germany (2006).

21. Canevarolo, S. V. - "Técnicas de Caracterização de Polímeros", Artliber, São Paulo (2004).

22. Marinho, J. R. D. - "Macromoléculas e Polímeros", Manole, Barueri (2005).

23. Goethals, E. J. - "Cationic Polymerization and related processes", in: 6th International Symposium Cationic Polymerization and related processes, Ghent, Belgium (1984).

24. Lucas, E. F.; Soares, B. G. \& Monteiro, E. - "Caracterização de Polímeros: Determinação de Peso Molecular e Análise Térmica", E-Papers, Rio de Janeiro (2001).

25. Mano, E. B. - "Polímeros Como Materiais de Engenharia", Edgard Blücher, Rio de Janeiro (1991).

26. Mohan, Y. M. \& Raju, K. M. - Int. J. Polym. Mater., 55, 3, p.203 (2006). http://dx.doi.org/10.1080/009140390925134

Enviado: 18/02/11

Reenviado: 08/11/11

Aceito: 09/11/11

Apêndice 1. Notação.

$\begin{array}{ll}\text { GAP } & \text { poli (metil azoteto de glicidila) } \\ \text { ECH } & \text { epicloridrina } \\ \text { PBLH } & \text { polibutadieno líquido com terminação hidroxílica } \\ \text { RDX } & \text { Research Department Explosive, ou ciclotrimetilenotrinitramina }\end{array}$

